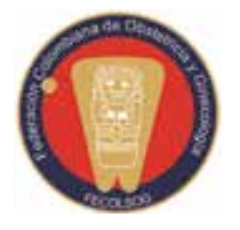

\title{
EDITORIAL
}

\section{GUIIAS DE PRÁCTICA CLÍNICA EN COLOMBIA}

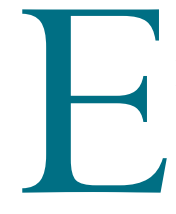

n el presente número continuamos con la publicación de las Guías de Práctica Clínica (GPC) para el Sistema General de Seguridad Social en Salud de Colombia. La versión publicada corresponde a una adaptación en formato de revista médica de acuerdo con las normas internacionales para este tipo de publicaciones (1). Las versiones completas y cortas fueron publicadas recientemente por el Ministerio de Salud y Protección Social (2).

Las GPC se definen como un conjunto de recomendaciones desarrolladas de forma sistemática para ayudar a profesionales y pacientes a tomar decisiones sobre la atención sanitaria más apropiada, y a seleccionar las opciones diagnósticas o terapéuticas más adecuadas a la hora de abordar un problema de salud o una condición clínica específica (3).

Las GPC se empiezan a producir entre los años cincuenta y setenta, con un importante incremento a partir de los años ochenta que finaliza con el mandato de la Agency for Health Care Research and Quality (AHRQ) de producir guías clínicas en Estados Unidos (4). En la actualidad se han convertido en una propuesta de los sistemas de salud para mejorar la calidad, disminuir la heterogeneidad de la atención médica y hacer más organizado y eficiente el manejo de los recursos $(5,6)$. También surgen de la comunidad médica, en un intento por preservar la autonomía en un medio cada vez más restrictivo por las presiones de los entes financiadores (7). En los últimos años, muchos países han acumulado gran experiencia en el desarrollo, la evaluación e implementación de guías de práctica clínica en ámbitos profesionales, institucionales, regionales y nacionales, reconociendo que las guías son una herramienta clave para mejorar la calidad y pertinencia de los servicios de salud (8-10). El consenso necesario para elaborar guías fue posible gracias a nuevas técnicas como el método Delphi (11) y los ensayos clínicos controlados que se convirtieron progresivamente en el estándar de oro para la evaluación de terapias (12), y las metodologías que fundamentan la aproximación de la medicina basada en las pruebas, tales como la definición de preguntas contestables, las búsquedas sistemáticas y la aproximación crítica a la calidad de la literatura médica (13).

Colombia inició su carrera hacia las guías a inicios de los años noventa, cuando el desaparecido Instituto de Seguros Sociales publicó las Guías de práctica clínica que fueron realizadas en asocio con la Universidad Javeriana (14). Posteriormente, en 1998, el Acuerdo 117 establece el obligatorio cumplimiento de las actividades, los procedimientos y las intervenciones de demanda inducida, así como la atención de enfermedades de interés en salud pública. Además, reglamenta y ordena la expedición de las Normas y Guías de Atención de las Acciones del Plan Obligatorio de Salud (POS) (15). Como consecuencia de esto aparece la convocatoria para desarrollar las Normas Técnicas de Protección Específica y Detección Temprana, así como las guías para enfermedades de interes en salud pública. Estas deberían obedecer a: alta frecuencia y severidad de carga social y económica, ser transmisibles o tener altas externalidades que afectaran rápidamente la salud, y que pudieran evitarse con intervenciones de baja complejidad que generaran un impacto positivo. Así nace la Resolución 412 de 2000 (16), que tuvo una metodología mixta. La gran mayoría de normas y guías tuvieron un gran peso de consenso de expertos, pero la de anticoncepción fue la única que adaptó una guía existente de la Organi- 
zación Mundial de la Salud (OMS). Las normas de la Resolución 412 de 2000 establecieron las actividades, los procedimientos y las intervenciones costo-efectivas de obligatorio cumplimiento por desarrollar en forma secuencial y sistemática en la población, y contenían las frecuencias anuales mínimas, así como los profesionales de salud a cargo. Las guías no tenían obligatorio cumplimiento, lo que creó confusión en el país, pues sí era obligatorio cubrir las patologías mas no necesariamente con las actividades planteadas en las guías, a excepción de los esquemas terapéuticos contemplados para lepra, malaria, tuberculosis y leishmaniasis cutánea y visceral. La mencionada Resolución tuvo participación asimétrica de actores del sistema de salud con influencia fundamental de expertos, no incluyó revisión por pares ni estudios piloto pero tuvo una formulación nacional. Ha tenido muchos obstáculos para su aplicación por nivel de atención, distribución geográfica, insuficiencia de recursos, dificultades técnicas y administrativas, así como pobre adherencia de los responsables. A estas dificultades en la implementación se suma que no hubo una versión única de contenidos, los flujogramas no eran claros, no hubo reconocimiento de las excepciones o circunstancias especiales, ni reconocimiento de aspectos locales o regionales.

En el año 2005 el Ministerio hace una convocatoria para actualizar algunas normas y guías de la Resolución 412 del 2000, y las relacionadas con Salud Sexual y Reproductiva en su mayoría quedan a cargo de la Universidad Nacional de Colombia. En esta ocasión el grupo desarrollador de la Universidad Nacional de Colombia utiliza una metodología verificable y repetible a fin de obtener la evidencia disponible en la literatura internacional para su evaluación crítica y para la construcción de las recomendaciones. Lamentablemente, este esfuerzo de actualizar la Resolución 412 no dio el paso de elevar a categoría de normas este producto, el cual ve la luz en una publicación de mayo del 2007 que hace el Programa de Apoyo a la Reforma en Salud (PARS) como: "Guías de promoción de la salud y prevención de enfermedades en la salud pública” (17). Esto nuevamente crea confusión en el país pues lo de obligatorio cumplimiento es la norma y aunque muchas de ellas están actualizadas, no se surte el proceso de pasar de guía a norma y siguen vigentes las de la 412 del 2000. En el año 2006 el Instituto Nacional de Cancerología, con el apoyo del Instituto de Investigaciones Clínicas de la Universidad Nacional, publica las guías de Cáncer de mama, Tamización del cáncer de cérvix y Vacunación en pacientes con cáncer (18). En el año 2008 la Universidad Nacional actualiza las normas de Complicaciones hemorrágicas e hipertensivas del embarazo, y la Universidad de Antioquia las de la sífilis congénita, en convocatoria del Ministerio de la Protección Social a través del Fondo de Población de las Naciones Unidas (UNPFA); sin embargo, estas guías no fueron nunca publicadas.

El trabajo que se presenta en este número de la Revista Colombiana de Obstetricia y Ginecología es el fruto de un esfuerzo sostenido y solidario de algunos de los grupos universitarios más importantes de Colombia: la Universidad de Antioquia, la Pontificia Universidad Javeriana y la Universidad Nacional de Colombia reunidos en torno de un proyecto común: el Centro de Investigaciones en Evaluaciones de Tecnologías en Salud (Cinets), que pretende brindarle al país un análisis serio, riguroso y académico de las más recientes tecnologías disponibles en salud, desde la perspectiva de su eficacia y la racionalidad de su costo mediante análisis económicos de costo-efectividad o costo-utilidad. Ello ha sido posible gracias a la decisión conjunta de las Universidades de incluir en sus agendas de investigación y extensión los más sentidos problemas de salud para la población colombiana, y abordarlos desde una perspectiva crítica, libre de sesgos y presiones externas, para adelantar estudios técnicos que contribuyan a un mejor actuar por parte de los responsables de las decisiones en salud.

La disponibilidad de "Guías de práctica clínica para la prevención, detección temprana y tratamiento de las alteraciones del embarazo, parto o puerperio" implica, entre otras cosas, estandarizar para Colombia el cuidado prenatal, enfatizando en la prevención, 
detección temprana y tratamiento oportuno de las alteraciones que afectan la gestación en todos los niveles de atención, buscando reducir la morbimortalidad materna asociada con el propósito de mejorar la salud materna y la calidad de la atención médica en todos los niveles de atención obstétrica. Se espera que las Guías Clínicas del Ministerio de Salud y Protección Social sean la referencia para la aplicación de procesos asistenciales en instituciones de primer, segundo y tercer nivel de atención. Las declaraciones contenidas para cada proceso reflejan la evidencia científica utilizada al tiempo que eran preparadas.

El vertiginoso avance de la ciencia, la explosión tecnológica, la globalización del conocimiento y las nuevas tendencias que ofrecen los tratados de libre comercio deben convertirse en instrumentos de desarrollo, de progreso social y de mejoramiento continuo de las condiciones de bienestar de las comunidades, dentro de los principios de equidad, respeto, justicia y calidad. En el sector de la salud, estos avances y sus enormes posibilidades corren paralelos con riesgos importantes que amenazan los principios enunciados, como son los intereses económicos de grupos monopólicos, la introducción inconsulta de tecnologías sin la satisfacción plena de los requisitos de utilidad y racionalidad económica y, fundamentalmente, las consideraciones de costo-oportunidad.

Este trabajo es una magnífica oportunidad para reivindicar el papel de la academia, de los grupos profesionales, de las sociedades científicas reunidas en torno de las Universidades como gestoras de conocimiento, de cambio social, de bienestar con respeto total por los derechos que asisten a las personas enfermas de cualquier sociedad moderna para acceder a servicios oportunos, eficaces y de alta calidad para la promoción, el sostenimiento o la recuperación de la salud en condiciones igualitarias.

Las Guías en Práctica Clínica son tan solo una herramienta del sistema de garantías de la calidad, pero su adecuado desarrollo permite poner a disposición de los pacientes -independientemente de su rango social, ubicación geográfica, condición económica u otras variables- una atención pronta, eficiente y dentro de los más altos estándares de calidad, de conformidad con el mejor conocimiento disponible derivado tanto de la investigación de punta como de la experiencia recogida por la tradición médica en el ejercicio de las buenas prácticas.

Ahora el país tiene un reto muy grande que va más allá de elevar estas guías a categoría de normas para que sean de obligatorio cumplimiento en el sistema, dicho reto tiene que ver con la implementación. Este es un proceso más largo y complejo, que implica la transmisión del conocimiento a los tomadores de decisiones y que se traduce en el uso de la mejor evidencia científica disponible según cada escenario clínico. La experiencia foránea ha mostrado que la implementación y adherencia a las recomendaciones es baja, y que las GPC son un recurso subutilizado. Esperamos que las estrategias planteadas por los grupos desarrolladores al Ministerio de Salud y Protección Social se cumplan y que en Colombia la implementación sí sea una realidad.

\section{Pio Iván Gómez-Sánchez, MD, MSc, Facog Editor invitado}

Profesor Titular, director del Grupo de Investigación en Salud Sexual y Reproductiva, Universidad Nacional de Colombia. Investigador principal-líder de la "Guía para la prevención, detección temprana y tratamiento de las complicaciones del embarazo, parto y puerperio". Miembro Academia Nacional de Medicina.

Hernando Gaitán Duarte, MD, MSc.

Editor

\section{REFERENCIAS}

1. International Committee of Medical Journal Editors. Recommendations for the Conduct, Reporting, Editing, and Publication of Scholarly Work in Medical Journals; 2013. [Visitado 2013 Sept 24]. Disponible en http://www.icmje.org/index.html 
2. Ministerio de Salud y Protección Social República de Colombia. Guías de Práctica Clínica; 2013. [Visitado 2013 Sept 24]. Disponible en: http://gpc.minsalud.gov. co/Pages/Default.aspx

3. Field MJ, Lohr KN, editors. Clinical Practice Guidelines: Directions for a New Program, Institute of Medicine. Washington, DC: National Academy Press; 1990.

4. Weisz G, Cambrosio A, Keating P, Knaapen L, Schlich T, Tournay VJ. The emergence of clinical practice guidelines. Milbank Q. 2007;85:691-72.

5. Open Clinical. Clinical Practice Guidelines. [Visitado 2013 Sept 24]. Disponible en: http://www.openclinical. org/guidelines.html

6. Fowkes FG, Roberts CJ. Introducing Guidelines into Clinical Practice. Eff Health Care. 1984;1:313-23.

7. Timmermans S, Kolker ES. Evidence-Based Medicine and the Reconfiguration of Medical Knowledge. Journal of Health and Social Behavior. 2004;45 Suppl:177-93.

8. Guía Salud. Guías de Práctica Clínica [Visitado 2013 Sept 24]. Disponible en: http://www.guiasalud.es/web/ guest/guias-practica-clinica

9. National Institute for Clinical Excellence. NICE Guidance. [Visitado 2013 Sept 24]. Disponible en: http://www.nice.org.uk/guidance/

10. National Health and Medical Research Council. Clinical Practice Guidelines Portal. [Visitado 2013 Sept 24]. Disponible en: http://www.clinicalguidelines.gov.au/
11. Linstone HA, Turoff M. The Delphi Method: Techniques and Applications, Reading: AddisonWesley; 1975.

12. Sackett DL, Haynes RB, Guyatt GH, Tugwell P. Clinical epidemiology: a basic science for clinical medicine. 2nd ed. Boston: Little Brown; 1991.

13. Davidoff F, Haynes B, Sackett D, Smith R. Evidence based medicine. BMJ. 1995;310:1085-6.

14. Instituto de Seguros Sociales. Guías de Práctica Clínica. Bogotá: ISS; [visitado 2013 Sept 24]. Disponible en: http:// www.iss.gov.co/portal/index.jsp? cargaHome $=508 \mathrm{id}$ categoria $=103 \&$ id_subcategoria $=1138$ id_seccion $=831$.

15. Ministerio de Salud Colombia. Acuerdo 117. [Visitado 2013 Sept 24]. Disponible en: http://www.minsalud. gov.co/Normatividad/ACUERDO\%20117\%20DE\%20 1998.pdf

16. Ministerio de Salud. República de Colombia. Resolución 412 de 2000.

17. Ministerio de la Protección Social. Programa de apoyo a la Reforma. Guías de promoción de la salud y prevención de enfermedades en la salud pública. Bogotá: Ministerio de la Protección Social.

18. Instituto Nacional de Cancerología. Recomendaciones y Guías. [Visitado 2013 Sept 24]. Disponible en: http://www.cancer.gov.co/contenido/contenido. aspx? catID $=-1 \& \operatorname{con} I D=766$ 